\section{Reintubación postoperatoria en pacientes sometidos a reemplazo valvular aórtico: extubación estándar vs ultra fast-track}

\author{
Postoperative reintubation in patients undergoing aortic valve \\ replacement: standard extubation vs ultra fast-track
}

Dra. Janaí Santiago-López,* Dr. Víctor León-Ramírez,*
Dra. Hilen Bravo-Briseño, ${ }^{\ddagger}$ Dr. Antonio Castellanos-Olivares
§

Citar como: Santiago-López J, León-Ramírez V, Bravo-Briseño H, Castellanos-Olivares A. Reintubación postoperatoria en pacientes sometidos a reemplazo valvular aórtico: extubación estándar vs ultra fast-track. Rev Mex Anestesiol. 2021; 44 (4): 245-249. https://dx.doi.org/10.35366/100868

RESUMEN. Introducción: Existe discrepancia para la extubación temprana de los pacientes sometidos a reemplazo valvular aórtico por estenosis, debido a su tendencia a desarrollar hipertensión postoperatoria que puede condicionar un fracaso del tratamiento quirúrgico. Objetivo: Comparar la incidencia de reintubación postoperatoria por hipertensión arterial en pacientes sometidos a reemplazo valvular aórtico con la técnica de extubación estándar vs ultra fast-track. Material y métodos: Se realizó un estudio de cohortes retrospectivo, incluyó a 73 pacientes sometidos a reemplazo valvular aórtico, se asignaron a dos grupos dependiendo de la técnica de extubación: estándar (grupo I) y ultra fast-track (grupo II). Se evaluó la presencia de hipertensión arterial y de reintubación postoperatoria. El análisis de variables se realizó con $\chi^{2}$. Una $p<0.05$ fue significativa. El procesamiento se realizó con el software SPSS v-24.0. Resultados: Cuarenta y cinco pacientes pertenecían al grupo I y 28 al grupo II. La incidencia de hipertensión arterial y de reintubación fue mayor para el grupo II ( $p=0.027)$, no hubo diferencias en cuanto a la morbilidad $(p=0.348)$, mortalidad ( $p$ $=0.202)$ y días de estancia postoperatoria $(p=0.182)$. Conclusión: La incidencia de reintubación postoperatoria por hipertensión arterial en pacientes sometidos a reemplazo valvular aórtico con la técnica ultra fast-track es mayor que con la extubación estándar en nuestra unidad hospitalaria.

ABSTRACT. Introduction: There is controversy about the use of early extubation in patients undergoing aortic valve replacement due to stenosis, given their tendency to develop postoperative hypertension, which could defeat the whole purpose of the surgical intervention. Objective: To compare the incidence of postoperative reintubation for hypertension in patients undergoing aortic valve replacement using the standard extubation technique and the ultra fast-track. Material and methods: A retrospective cohort study that included 73 cases of patients undergoing aortic valve replacement. The patients were divided into two according to the extubation technique used: standard (group I) and ultra fast-track (group II). The presence of hypertension and postoperative reintubation was evaluated. The analysis of variables was performed using the $\chi^{2}$ test. A $p<0.05$ was considered significant. The data were processed using SPSS v. 24.0. Results: 45 patients were in group I and 28 in group II. The incidence of hypertension and reintubation was higher in group II $(p=0.027)$. There were no differences in morbidity $(p=0.348)$, mortality $(p=0.202)$ and length of postoperative stay $(p=0.182)$. Conclusion: In our hospital unit, the incidence of postoperative reintubation for hypertension in patients undergoing aortic valve replacement with the ultra fast-track technique is higher than with standard extubation.

\section{INTRODUCCIÓN}

L a hipertensión arterial es una manifestación frecuente en el postoperatorio de la cirugía cardíaca con derivación cardiopulmonar, y es descrito por algunos autores como una de las fases «fisiológicas» en la restauración de la estabilidad hemodinámica posterior a la mis$\mathrm{ma}^{(1)}$. Por lo general, se asocia con taquicardia, ambas originadas por la descarga adrenérgica que sigue a la resolución del efecto anestésico, así como a factores intra- y postoperatorios (catecolaminas endógenas, aumento de la reactividad vascular, etcétera). Cabe señalar que el grupo de pacientes previamente hipertensos será más susceptible a este fenómeno.

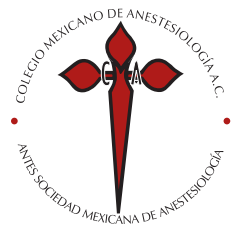

Palabras clave: Cirugía cardíaca, extubación inmediata, hipertensión, morbilidad.

Keywords:

Heart surgery, tracheal extubation, hypertension, morbidity.

\footnotetext{
* Departamento de Anestesiología, Hospital de Cardiología.

‡ Departamento de Anestesiología, Hospital de Especialidades «Dr. Bernardo Sepúlveda». $\S$ Jefatura del Departamento de Anestesiología. Hospital de Especialidades «Dr. Bernardo Sepúlveda».
}

Centro Médico Nacional Siglo XXI del Instituto Mexicano del Seguro Social. Ciudad de México.

Correspondencia:

Janaí Santiago-López

Hospital de Cardiología del Centro Médico Nacional Siglo XXI, Avenida Cuauhtémoc 330, Col. Doctores, Alcaldía Cuauhtémoc, Ciudad de México. C.P. 06720. Tel. 56-27-69-00 ext. 22181. E-mail: janai_santiago@ yahoo.com.mx

Recibido: 12-03-2020 Aceptado: 26-11-2020 
Si bien es imprescindible mantener una presión arterial media adecuada para la perfusión de los órganos, especialmente el miocardio, la hipertensión arterial debe ser tratada enérgicamente. Hay grupos de patología que en particular tienden a desarrollar hipertensión arterial severa, en los cuales su control debe ser aún más preciso, como es el caso de la sustitución valvular por estenosis aórtica. En este caso, suele existir una importante hipertrofia ventricular izquierda que, junto a unas resistencias vasculares elevadas, conduce al desarrollo de hipertensión arterial severa ${ }^{(2,3)}$.

La hipertensión arterial puede comprometer las suturas vasculares, especialmente las realizadas en la aorta, y disminuye el flujo diastólico coronario por el aumento de la postcarga, lo cual constituye un importante factor de riesgo de mortalidad temprana, por lo que estos pacientes deben ser hospitalizados en una Unidad de Cuidados Intensivos con monitoreo electrocardiográfico y de la presión arterial invasiva, además de hacerse un tratamiento enérgico del dolor y la ansiedad ${ }^{(1,4-6)}$.

La presión arterial y la velocidad de eyección del ventrículo izquierdo son los dos factores más importantes en la evolución de los pacientes postoperados de reemplazo valvular aórtico por estenosis. Por lo tanto, el tratamiento médico debe ir orientado a disminuir estos factores, lo que se consigue con sedoanalgesia bajo intubación endotraqueal. Un control inadecuado del dolor y de la presión arterial puede representar un fracaso del tratamiento quirúrgico ${ }^{(6,7)}$. Sin embargo, los programas de recuperación postoperatoria rápida en cirugía cardíaca marcan una tendencia reciente hacia la simplificación de los cuidados postoperatorios; en este sentido, la extubación postoperatoria inmediata constituye una de las premisas más discutidas de este modelo, generando un interés creciente ${ }^{(8-21)}$.

Aún existe una discrepancia frente a la decisión de extubar a un paciente en el quirófano después de una cirugía cardíaca. En especial, para el caso de reemplazo valvular aórtico por estenosis.

\section{MATERIAL Y MÉTODOS}

Con la aprobación de la Comisión de Investigación Científica del hospital y con el objeto de comparar la incidencia de reintubación postoperatoria por hipertensión arterial severa en pacientes sometidos a reemplazo valvular aórtico con la técnica de extubación estándar vs ultra fast-track en nuestra unidad hospitalaria, se realizó un estudio de cohortes retrospectivo que incluyó a 73 pacientes.

Para la obtención del listado de pacientes se llevó a cabo una revisión de los archivos de movimiento hospitalario. Se tomó nombre y número de seguridad social de los pacientes que fueron sometidos a reemplazo valvular aórtico y fueron revisados en el expediente electrónico Intracard ${ }^{\circledR}$, se identificaron aquellos pacientes cuyo diagnóstico al ingreso fue estenosis aórtica crítica, se confirmó el nombre y número de seguridad social. De igual forma, se revisaron las copias de los informes operatorios del Servicio de Cirugía Cardiotorácica. De esta manera, se confeccionó un listado inicial de 104 pacientes.

Posteriormente, se procedió a la solicitud de los expedientes clínicos en el departamento de archivo y se les aplicaron los criterios de inclusión, exclusión y de eliminación. Se incluyeron pacientes de cualquier sexo, mayores de edad, con riesgo anestésico-cardíaco CARE I-IV. Se excluyeron aquellos pacientes que al momento de la recolección de datos no se localizaron sus expedientes. El criterio de eliminación fue aquellos con alguna carencia estructural en el expediente clínico que impidiera el llenado completo de la hoja de recolección de datos. Se seleccionó así una serie definitiva de 73 pacientes.

Una vez elaborada, se obtuvo de cada paciente toda la información necesaria. La técnica de extubación se determinó en función de la temporalidad de la extubación, se consideró extubación estándar si se realizaba en la Unidad de Terapia Postquirúrgica y ultra fast-track si se realizaba en el quirófano. Mientras que la reintubación postoperatoria

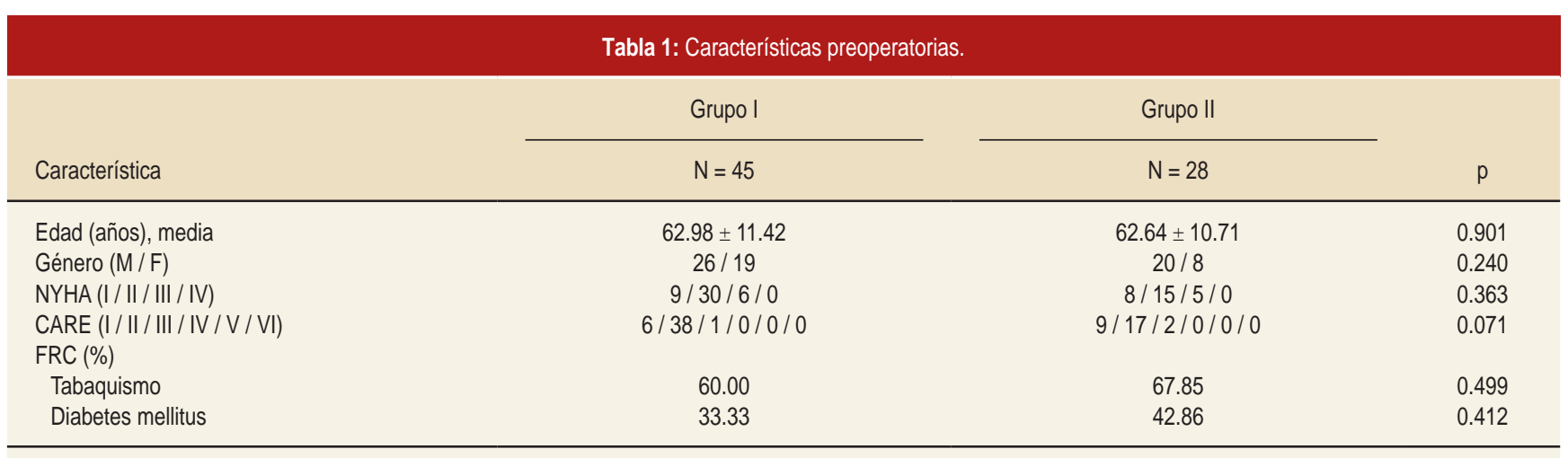

M = masculino; F = femenino; NYHA = escala New York Heart Association; CARE = riesgo anestésico cardíaco; FRC = factores de riesgo cardiovascular. 


\begin{tabular}{|c|c|c|c|}
\hline \multirow[b]{2}{*}{ Característica } & Grupo I & Grupo II & \multirow[b]{2}{*}{$p$} \\
\hline & $\begin{array}{c}N=45 \\
\text { Media } \pm D E\end{array}$ & $\begin{array}{c}N=28 \\
\text { Media } \pm D E\end{array}$ & \\
\hline Tiempo anestésico (min) & $270.53 \pm 69.64$ & $250.36 \pm 70.04$ & 0.234 \\
\hline Tiempo quirúrgico (min) & $228.76 \pm 62.53$ & $216.36 \pm 62.94$ & 0.414 \\
\hline Tiempo de CEC (min) & $107.49 \pm 53.37$ & $126.46 \pm 71.88$ & 0.201 \\
\hline Tiempo de PAo (min) & $94.33 \pm 67.73$ & $114.96 \pm 85.11$ & 0.256 \\
\hline Tipo de prótesis (M / B) & $18 / 27$ & $10 / 18$ & 0.989 \\
\hline
\end{tabular}

CEC = circulación extracorpórea; $\mathrm{PAo}=$ pinzamiento aórtico; $\mathrm{M}$ = mecánica; $\mathrm{B}$ = biológica.

por hipertensión arterial severa se consideró cuando una vez extubado el paciente hubo la necesidad de reintroducirle un tubo endotraqueal por presentar elevación sostenida de las cifras tensionales por arriba de 180/110 a pesar del tratamiento antihipertensivo habitual a base de una perfusión intravenosa de nitrovasodilatadores, betabloqueadores y liberadores de óxido nítrico, además de una volemia adecuada y el uso racional de las aminas. Los datos obtenidos fueron recogidos en un formulario de fácil aplicación, con pruebas diseñadas para tal fin. El proceso de identificación de expedientes de pacientes y la recolección de datos fue realizado por el residente de anestesiología.

Para el análisis de la información se utilizó estadística de tipo descriptivo y medidas de asociación. Para la evaluación de las variables demográficas se utilizó estadística descriptiva con medidas de tendencia central y dispersión para las variables continuas y proporciones para las variables nominales y categóricas.

Para analizar las diferencias entre los grupos se utilizó $\chi^{2}$. Otras comparaciones entre variables numéricas se realizaron con la prueba t de Student. Un valor de $\mathrm{p}<0.05$ fue considerado estadísticamente significativo.

Para el procesamiento y análisis estadístico de los datos se construyó una base de datos electrónica con el software SPSS versión 24.0 para Windows (SPSS Inc., Chicago, IL, EUA).

\section{RESULTADOS}

Se incluyeron 73 pacientes en el estudio; 45 pacientes se extubaron mediante la técnica estándar (grupo I) y 28 mediante la técnica ultra fast-track (grupo II). Se analizaron las variables demográficas y las condiciones médicas preoperatorias y no hubo diferencias significativas entre ambos grupos (Tabla 1).

Las características intraoperatorias tampoco mostraron diferencias (Tabla 2).

Las complicaciones postoperatorias se presentaron en 19 pacientes $(26.03 \%)$, la complicación más frecuente fue el sangrado mayor a lo habitual en $9.56 \%$ de los casos. Sólo un paciente (1.37\%) falleció durante el período postoperatorio. No se encontraron diferencias significativas en cuanto a la morbimortalidad postoperatoria.

Se encontró una diferencia significativa en la reintubación por hipertensión arterial severa, siendo mayor en el grupo II ( $p=0.027)$. La duración media de la estancia en la Unidad de Terapia Postquirúrgica fue de 17.26 + 10.66 días sin mostrar diferencias significativas entre ambos grupos $(\mathrm{p}=0.182)$ (Tabla 3).

\section{DISCUSIÓN}

El auge de la cirugía cardíaca ha originado un interés creciente en los proyectos de recuperación postoperatoria facilitada, marcando una tendencia hacia la simplificación de los cuidados postoperatorios, sin inmolar la seguridad del paciente o los resultados quirúrgicos.

Bajo esta acepción, las técnicas de extubación temprana (ultra fast-track y fast-track) constituyen uno de los supuestos más discutidos de este arquetipo. Aunque pocos grupos quirúrgicos utilizan dichas técnicas, cada vez más se publican artículos que pretenden explorar su seguridad(8-18).

Sin duda, los resultados de estudios de pacientes sometidos a cirugía cardíaca con derivación cardiopulmonar extubados con técnica ultra fast-track son escasos; y en lo que a la tasa de extubación se refiere, nuestros resultados parecen ser más austeros que los reportados en estas publicaciones. Lo que coincide con el enfoque conservador de Reis $\mathrm{J}$ y colaboradores, quienes extubaron a $42 \%$ de cirugías con derivación cardiopulmonar dentro de la hora de finalizada la misma ${ }^{(18)}$, en nuestro estudio tan sólo se lograron extubar a $38.36 \%$ de los pacientes, en comparación con algunos autores que han logrado extubar en el quirófano de 87-100\% de los pacientes ${ }^{(12-20,22)}$.

Esto puede ser atribuido a que en nuestra institución hospitalaria no existen protocolos de extubación temprana (ultra fast-track y/o fast-track), por lo que la decisión en cuanto al momento de la extubación está determinada principalmente 
por la preferencia del anestesiólogo, quienes en su mayoría muestran una perspectiva tradicionalista.

Gangopadhyay S y colegas reportan que la extubación fue posible en $88 \%$ de los pacientes sometidos a cirugía cardíaca con derivación cardiopulmonar y ninguno de ellos requirió de reintubación por insuficiencia respiratoria, siendo comparables los parámetros hemodinámicos y las complicaciones postoperatorias en comparación con el grupo de extubación convencional $^{(22)}$.

Hemmerling TM y colaboradores reportaron extubar a $100 \%$ de los pacientes sometidos a cirugía valvular aórtica dentro de los 20 minutos de concluido el evento, a pesar de que algunos de ellos requirieron el apoyo de marcapasos ${ }^{(12)}$.

Desde el punto de vista de la seguridad de la técnica de extubación temprana en cirugía cardíaca, se destaca que la tasa de reintubación endotraqueal oscila alrededor de $2 \%$ en un rango de $0.7-7.7 \%^{(15)}$. En nuestro estudio hubo una necesidad de reintubación de $9.59 \%$, muy por encima de lo reportado en la literatura internacional.

Esto podría deberse a que en la mayoría de las publicaciones que evaluaron el modelo de ultra fast-track con extubación inmediata en el quirófano después de la cirugía cardíaca incluyeron el uso de analgesia epidural torácica para el control postoperatorio del dolor y en otros casos se incluyó el uso de analgesia postoperatoria con remifentanilo, prácticas no incluidas en el nuestro ${ }^{(17,22,23)}$.

La analgesia epidural o intravenosa es un complemento importante de la técnica de extubación temprana después de la cirugía cardíaca bajo derivación cardiopulmonar, ya que bloquea la respuesta simpática, optimiza la mecánica ventilatoria, los pacientes permanecen despiertos y es posible una movilización temprana por ausencia de dolor, lo que repercute en la restauración de la función fisiológica del paciente ${ }^{(22,23)}$.

Otro hallazgo encontrado en nuestra cohorte es que el uso de la técnica de extubación ultra fast-track tiene riesgos similares de mortalidad y complicaciones postoperatorias mayores a la extubación convencional y, aunque esta intervención reduce el tiempo de extubación, no reduce la duración de la estancia intrahospitalaria, lo que coincide con lo reportado por los autores Zhu F y Wong WT con sus respectivos colaboradores en sus revisiones sistemáticas ${ }^{(23,24)}$.

Consideramos que los resultados obtenidos de este estudio podrían servir como una guía frente a la toma de decisión de extubar un paciente en el quirófano después de una sustitución valvular aórtica, en especial en los casos de estenosis aórtica crítica. La restricción de extubar a aquellos pacientes que presentan esta condición clínica en particular, sin duda alguna disminuirá la tasa de extubados con técnica ultra fast-track, pero a su vez se reducirá la necesidad de reintubación durante el período postoperatorio y se incrementará la confianza en el método por parte de los servicios de apoyo.

$\mathrm{Y}$ aunque en nuestro estudio no encontramos diferencias estadísticamente significativas en cuanto a las características preoperatorias y transoperatorias, consideramos deben ser evaluados y reevaluados en futuros ensayos clínicos aleatorizados aquellos factores de riesgo adicionales referidos en la literatura internacional como la edad avanzada, la cirugía de urgencia, obesidad, antecedente de enfermedad pulmonar, hipertensión descontrolada preoperatoria, afección concomitante en el territorio de la arteria coronaria derecha (sobre todo el de la descendente posterior), antecedente de insuficiencia cardíaca y/o disfunción ventricular, inestabilidad hemodinámica sostenida transoperatoria, requerimiento de marcapasos (por bloqueo auriculoventricular $[\mathrm{A}-\mathrm{V}]$ ), sangrado, hipovolemia, pinzamiento aórtico mayor a 90 minutos, dificultades a la salida de bomba, tasa de opioide y temperatura transderivación cardiopulmonar $^{(8-25)}$.

\section{CONCLUSIÓN}

La incidencia de reintubación postoperatoria por hipertensión arterial severa en pacientes sometidos a reemplazo valvular aórtico con la técnica ultra fast-track es mayor que con la extubación estándar en nuestra unidad hospitalaria.

\begin{tabular}{lcc} 
& Tabla 3: Características postoperatorias. & \\
& Grupo I & Grupo II \\
\cline { 2 - 3 } Característica & $\mathrm{N}=45$ & $\mathrm{~N}=28$ \\
\hline Reintubación HAS (Sí / No) & $7 / 21$ & $3 / 42$ \\
Reintubación otras causas (Sí / No) & $5 / 41$ & $4 / 23$ \\
Morbilidad (Sí / No) & $9 / 19$ & $10 / 35$ \\
Mortalidad (Sí / No) & $1 / 27$ & $0 / 45$ \\
Estancia UTPQ (días), media & $15.14 \pm 5.00$ & $18.58 \pm 12.8$ \\
\hline HAS = hipertensión arterial sistémica; UTPQ = Unidad de Terapia Postquirúrgica. & $0.027^{*}$ \\
* Significancia estadística. & & 0.348 \\
\end{tabular}




\section{REFERENCIAS}

1. Principios de urgencias, emergencias y cuidados críticos. Capítulo 1. 15. Manejo del paciente en el postoperatorio de cirugía cardiaca. 5. Manejo de la hipertensión arterial en el postoperatorio de CEC. [Consultado 5 de mayo de 2016]. Disponible en: https://www.uninet.edu/tratado/c011505.html

2. Gosavi S, Channa R, Mukherjee D. Systemic hypertension in patients with aortic stenosis: clinical implications and principles of pharmacological therapy. Cardiovasc Hematol Agents Med Chem. 2015;13:50-53.

3. Capoulade R, Clavel MA, Mathieu P, Coté N, Dumesnil JG, Arsenault $\mathrm{M}$, et al. Impact of hypertension and renin-angiotensin system inhibitors in aortic stenosis. Eur J Clin Invest. 2013;43:1262-1272.

4. Betancourt-Rodríguez JI. Cuidado crítico cardiovascular: Manejo postoperatorio de cirugía cardiovascular del adulto. [Consultado 5 de febrero de 2020]. Disponible en: http://www.sld.cu/galerias/pdf/sitios/ anestesiologia/postop_en_cir_card..pdf

5. Reinthaler M, Stahli BE, Gopalamurugan AB, Xiu PY, Aggarwal SK, Frohlich G, et al. Post-procedural arterial hypertension: implications for clinical outcome after transcatheter aortic valve implantation. J Heart Valve Dis. 2014;23 675-682.

6. Sullivan BL. Con: early extubation in the operating room following cardiac surgery. Semin Cardiothorac Vasc Anesth. 2012;16:187-189.

7. Peláez-Romero R, Hortal FJ, Riesgo M. Tratamiento del dolor postoperatorio en cirugía cardíaca. Rev Esp Anestesiol Reanim. 2002;49:474-484.

8. Dayan R, Borracci RA, Rubio M, De Simon EA. La extubación inmediata en el quirófano como conducta de primera elección después de cirugía cardíaca en adultos. Rev Arg Anest. 2005;63:82-90.

9. Dorsa A, Rossi IA, Vrancic JM, Thierer J, Vaccarino GN, Piccinini F, et al. Extubación postoperatoria inmediata (ultra fast-track) en cirugía coronaria sin circulación extracorpórea. Rev Arg Anest. 2005;63:91-100.

10. Oxelbark S, Beningsson L, Eggersen M. Fast track as a routine for open heart surgery. Eur J Cardiothorac Surg. 2001;19:460-463.

11. Djaiani GN, Ali M, Heinrich L, Bruce J, Carroll J, Karski J, et al. Ultra fast-track anesthetic technique facilitates operating room extubation in patients undergoing off-pump coronary revascularization surgery. J Cardiothorac Vasc Anesth. 2001;15:152-157.

12. Hemmerling TM, Le N, Olivier JF, Choiniere JL, Basile F, Prieto I. Immediate extubation after aortic valve surgery using high thoracic epidural analgesia or opioid-based analgesia. J Cardiothorac Vasc Anesth. 2005;19:178-181.

13. Vricella LA, Dearani JA, Gundry SR, Razzouk AJ, Brauer SD, Bailey LL. Ultra fast-track in elective congenital cardiac surgery. Ann Thorac Surg. 2000;69:865-871.
14. Quasha AL, Loeber N, Feeley TW, Ullyot DJ, Roizen MF. Postoperative respiratory care: a controlled trial of early and late extubation following coronary artery bypass grafting. Anesthesiology. 1980;52:135-141.

15. Myles PS, Daly DJ, Djaiani G, Lee A, Cheng DC. A systematic review of the safety and effectiveness of fast-track cardiac anesthesia. Anesthesiology. 2003;99:982-987.

16. Hemmerling TM, Fortier JD, Basile I, Prieto F. Ultra fast track anesthesia in off-pump cardiac surgery: maintenance of core temperature is more important than specific anesthetic techniques. Can J Anesth. 2002;49:A47.

17. Straka Z, Brucek P, Vanek T, Votava J, Widimsky P. Routine immediate extubation for off-pump coronary artery bypass grafting without thoracic epidural analgesia. Ann Thorac Surg. 2002;74:1544-1547.

18. Reis J, Mota JC, Ponce P, Costa-Pereira A, Guerreiro M. Early extubation does not increase complication rates after coronary artery bypass graft surgery with cardiopulmonary bypass. Eur J Cardiothorac Surg. 2002;21:1026-1030.

19. Singh KE, Baum VC. Pro: early extubation in the operating room following cardiac surgery in adults. Semin Cardiothorac Vasc Anesth. 2012;16:182-186.

20. Badhwar V, Esper S, Brooks M, Mulukutla S, Hardison R, Mallios $D$, et al. Extubating in the operating room after adult cardiac surgery safely improves outcomes and lowers costs. J Thorac Cardiovasc Surg. 2014;148:3101-3109.

21. van Mastrigt GA, Maessen JG, Heijmans J, Severens JL, Prins MH. Does fast-track treatment lead to a decrease of intensive care unit and hospital length of stay in coronary artery bypass patients? A meta-regression of randomized clinical trials. Crit Care Med. 2006;34:1624-1634.

22. Gangopadhyay S, Acharjee A, Nayak SK, Dawn S, Piplai G, Gupta K. Immediate extubation versus standard postoperative ventilation: our experience in on pump open heart surgery. Indian J Anaesth. 2010;54:525-530.

23. Zhu F, Lee A, Chee YE. Fast-track cardiac care for adult cardiac surgical patients. Cochrane Database Syst Rev. 2012;10:CD003587. doi: 10.1002/14651858.CD003587.pub2.

24. Wong WT, Lai VK, Chee YE, Lee A. Fast-track cardiac care for adult cardiac surgical patients. Cochrane Database Syst Rev. 2016;9:CD003587.

25. Rodriguez-Blanco YF, Carvalho EM, Gologorsky A, Lo K, Salerno TA, Gologorsky E. Factors associated with safe extubation in the operating room after on-pump cardiac valve surgery. J Card Surg. 2016;31:274281. 